\title{
Studien über Pflanzenkolloide, XIII.
}

\section{Ueber synthetische Amylophosphorsäuren.}

\author{
Von M. Samec und Anka Mayer. \\ (Aus dem chemischen Institut der Universităt in Laibach.)
}

(Eingegangen am 19. Mărz 192\%)

Die seinerzeit von $M$. Sa me ${ }^{1}$ ) entwickelte Ansicht, daB der gallertige Anteil der Stärke, das Amylopektin, ein Kohlehydratphosphorsäureester sei, konnte in letzter Zeit durch die direkte Synthese verifiziert werden ${ }^{2}$ ).

Zur Durchführung derselben war es einerseits notwendig, das dem Amylopektin zugrunde liegende Kohlehydrat $\mathrm{zu}$ isolieren und anderseits die Veresterung so zu leiten, daß weder eine Hydrolyse noch eine Desaggregation des Polysaccharids stattfindet. Der erste Teil der Aufgabe konnte durch Verseifung des elektrodialytisch abgeschiedenen und von den Amylosen befreiten Amylopektins gelöst werden ${ }^{3}$ ), dem zweiten Teil wird unter besonderen Vorsichtsmabregeln die C. Neuberg'sche Phosphorylierungsmethode gerecht, mit deren Hilfe J. Kerb4) lösliche Stärke phosphorylieren konnte.

Eine synthetische Reaktion, an welcher ein so hochmolatiges Gebilde, wie die Erythroamylosen es sind, beteiligt ist, wird begreiflicherweise nach mehreren verschiedenen Richtungen verlaufen können und man hat a priori selbst bei gelungener Synthese nicht eine völlige Identität des nativen und synthetischen Produktes zu erwarten. In der Haupteigenschaft: dem Phosphorgehalte und der hohen Zähigkeit aber stimmen gewisse synthetische Amylophosphorsäuren mit der nativen völlig überein, so daB an dem kausalen Zusammenhang dieser beideń Eigenschaften nicht mehr gezweifelt werden kann.

1) M. S a mec u. F. v. Hoefft, Kolloidchem. Beih. 5, 141 (1913).

2) M. Samec u. Anka Mayer, Compt. rend. 173, 321 (1921).

3) Dieselben, Kolloidchem. Beih. 13, 272 (1921).

4) J. Kerb, Biochem. Zeitschr. 100, 3 (1919). 
In der vorliegenden Arbeit soll eine nähere Charakteristik synthetischer Amylophosphorsäuren versucht werden, welche auch im Hinblick auf die noch nicht geklärte Beziehung zwischen Amylo- und Erythrokörpern ${ }^{1}$ ) ein besonderes Interesse verdient.

I.

Die für die Phosphorylierung benötigten Erythroamylosen wurden durch sechsstündiges Erhitzen einer zweiprozentigen Amylopektinlösung auf $120^{\circ}$ dargestellt. Die Lossung enthält neben den Erythroamylosen noch mehr oder weniger zersetztes Amylopektin und gliedert sich bei der Elektrodialyse in mehrere verschieden viskose flüssige Phasen, welche nur schwer voneinander getrennt werden können.

Pipettiert man aus verschiedener Höhe der Elektrodialysierküvette Flüssigkeitsproben $a b$, so findet man vcn oben nach unten eine $\mathrm{Zu}$ nahme des Gehaltes der Lōsung an fester Substanz, einen Anstieg der spezifischen Leitfăhigkeit, ein Schwinden der blauen und Hervortreten der violetten Jodfarbe; in derselben Reihenfolge steigt die spezifische Leitfăhigkeit und die mittlere Molatgröße, sowie der Phosphorgehalt. Ausgenommen die Substanz der untersten Schichte bleiben alle Proben monatelang klar (Tabelle I).

Tabelle I.

Elektrodialytische Zerlegung einer 6 Sturiden auf $120^{\circ}$ erhitzten $z$ weiprozentigen Amylopektinlösung.

\begin{tabular}{|c|c|c|c|c|c|c|c|}
\hline $\begin{array}{l}\text { Tiefe der Schichte unter dem } \\
\text { Niveau, cm }\end{array}$ & 1 & 2 & 3 & 4 & 5 & 6 & 7 \\
\hline Trockensubstanz, Proz. . & 2,40 & 2,83 & 2,98 & 3,14 & 3,53 & 3,77 & 4,97 \\
\hline $\left.10^{5} \cdot \mathbf{k}^{*}\right) \cdot \cdot \quad \cdot \quad \cdot$ & 0,42 & 0,35 & 0,52 & 0,65 & \multicolumn{2}{|c|}{$\begin{array}{c}0,69 \mid 0,91 \\
\text { violett }\end{array}$} & 1,10 \\
\hline Jodfarbe $\frac{\text { wenig Jod }}{\text { überschüss. Jod }} \cdot \dot{ }$ & \multicolumn{2}{|c|}{ blau } & $\begin{array}{l}\text { viol. } \\
\text { blau } \\
\text { viol. }\end{array}$ & \multicolumn{4}{|c|}{ violett } \\
\hline Mittlere Molatgröße. & & & $13 \overline{6}$ & 000 & \multicolumn{2}{|c|}{173000} & \\
\hline $\begin{array}{c}\mathrm{P}_{2} \mathrm{O}_{5} \text { in Proz. der Trocken- } \\
\text { substanz } \\
\cdot\end{array}$ & \multicolumn{2}{|c|}{0,00} & \multicolumn{3}{|c|}{0,009} & \multicolumn{2}{|c|}{0,015} \\
\hline
\end{tabular}
( 2 bis $3.10^{-6}$ ).

Für die Phosphorylierung, welche nach den Angaben von J. Kerb durchgeführt wurde, wurden nur die obersten, so gut wie phosphorfreien Schichten verwendet. Nach beendeter Reaktion wurde das Gros

1) M. S a m e c, Kolloidchem. Beih. 10, 289 (1919). 
der Salze entfernt und die organische Substanz mit Alkohol gefällt. Das erhaltene klebrige Koagulum, welches genau dem nach $Z$. GruZewska abgeschiedenen Amylopektin gleicht, wurde mit 40 prozentigem Alkohol bis zum Verschwinden der $\mathrm{Cl}$-Reaktion gewaschen; es bildet mit Wasser eine undurchsichtige Suspension und ist auch in der Siedehitze nicht loslich. Während der Elektrodialyse wandern nachweislich $\mathrm{Ca}$-lonen in den Kathodenraum, an der Anodenmembran aber sondert sich eine durchsichtige, äußerst zähe Gailerte $a b$, welche äußerlich völlig mit dem aus dem Stärkekorn erhaltenen Amylopektin übereinstimmt. Nachdem die elektrische Leitfähigkeit des Sols konstant geworden war, wurde die Gallerte gesammelt. Ihre Eigenschaften verglichen mit denen der beiden Muttersubstanzen (Amylopektin und Erythroamylosen) enthält die Tabelle II.

Tabelle II.

\begin{tabular}{|c|c|c|c|c|c|c|c|}
\hline & 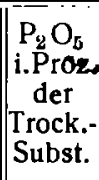 & $\begin{array}{l}\left.10^{5} . \mathrm{K}^{1}\right\rangle \\
\text { in } \\
\text { 2 proz. } \\
\text { Lơsg. }\end{array}$ & $\begin{array}{c}\mathrm{t} / \mathrm{t}_{1}{ }^{2}{ }^{1} \\
\text { in } \\
\text { p poroz. } \\
\text { Lösg. }\end{array}$ & $\begin{array}{l}\text { Jod- } \\
\text { farbe }\end{array}$ & $\begin{array}{c}\text { Mittlere } \\
\text { Molat- } \\
\text { groBe }\end{array}$ & $\begin{array}{c}\text { Dialysabler } \\
\text { Anteil } \\
\text { in Proz. } \\
\text { der gelöst. } \\
\text { Substanz }\end{array}$ & $\begin{array}{c}\text { Reduk- } \\
\text { tions- } \\
\text { ver- } \\
\text { mög. } \\
\end{array}$ \\
\hline $\begin{array}{c}\text { Natives Amylo- } \\
\text { pektin } .\end{array}$ & 0,175 & 8,21 & 14,96 & viol. & 113000 & 0,8 & 0,20 \\
\hline Erythroamylosen & 0,000 & 0,52 & 1,16 & $\begin{array}{l}\text { braun } \\
\text { viol. }\end{array}$ & 158800 & 17,56 & 1,26 \\
\hline $\begin{array}{l}\text { Phosphorylierte } \\
\text { Erythroamylosen }\end{array}$ & 2,190 & 29,21 & 17,7 & $\begin{array}{l}\text { braun } \\
\text { viol. }\end{array}$ & 62500 & 1,18 & 1,21 \\
\hline
\end{tabular}

1) Spez. Leitfähigkeit der Lösung nach Abzug des Wertes für destilliertes Wasser $\left(2-4.10^{-6}\right)$.

2) Vechăltnis der Durchlaufzeiten von Lösung und Wasser in einem Ostw a Id'schen Viskosimeter bei $25^{\circ} \mathrm{C}$.

3) Von $100 \mathrm{~g}$ Substan $z$ abgeschiedene Menge Kupfer.

Während beim nativen Amylopektin und den Erythroamylosen die osmotischen Steighöhen direkt eine Berechnung der mittleren Molatgröße ermöglichen, beteiligt sich bei den phosphorylierten Amylosen eine größere Menge von nicht dialysablen Ionen am osmotischen Druck. Ihr Einfluß läßt sich jedoch durch nachstehende Rechnung abschätzen.

Eine 0,98 prozentige Lösung des synthetischen Amylopektins übte einen osmotischen Druck von $65 \mathrm{~mm}$ Wassersäule aus. Die elektrische Leitfähigkeit des Osmometerinhaltes betrug $11,68 \cdot 10^{-5}$, die der AuBenflüssigkeit $1,61 \cdot 10^{-5}$. Die Konzentration der $\mathrm{H}$-lonen innen war 
gleich $4,9 \cdot 10^{-6} \mathrm{n}$ und in der AuBenflüssigkeit $0,6 \cdot 10^{-6} \mathrm{n}$. AuBer den $\mathrm{H}$-Ionen, deren Konzentration zu gering ist, um einen nennenswerten Anteil am osmotischen Druck zu bedingen, enthält die Lösung noch $\mathrm{Ca}-$ Ionen, Amylophosphat-Ionen und Neutralteile.

Die Konzentration der $\mathrm{Ca}$-Ionen können wir auf folgende Art ermitteln :

Die spezifische Leitfähigkeit des am osmotischen Druck beteiligten Anteiles beträgt nach obigen Messungen $10,07 \cdot 10^{-5}$, reziproke Ohm. Da im Hinblick auf die bei der Alkalistärke gemachten Erfahrungen das sekundäre Kalziumamylophosphat nicht lösungsstabil ist, dürfen wir mit großer Wahrscheinlichkeit in unserer sehr beständigen etwas sauren Gallerte nur das primäre $\mathrm{Salz}^{1}$ ) annehmen, so dab sich an der Stromleitung die Ionen $\mathrm{Ca}^{*}$ und $2\left(\mathrm{C}_{6} \mathrm{H}_{10} \mathrm{O}_{5}\right)_{\mathrm{x}} \mathrm{PO}_{4} \mathrm{H}$ beteiligen. Nohmen wir die mittlere Aequivalentwanderungsgeschwindigkeit der beteiligten Ionen mit 50 an, so würde die gemessene spezifische Leitfăhigkeit durch den Ausdruck 10,07.10-5 $=\frac{4 . x .50}{1000}$ gegeben sein, in welchem $x$ die Aequivalentkonzentration der Ionen bedeutet. Daraus. ist $x=0,000503 \mathrm{n}$. Die Aequivalentkonzentration des Ca-lons beträgt dann 0,000255 und die molekulare 0,000125. Dieser Menge von $\mathrm{Ca}$-lonen entspricht bei Vernachlässigung der Temperaturkorrektur ein osmotischer Druck 22,4 .0,0001252 . 7,60 . 13,6 =29 mm Wassersäule ${ }^{2}$ ). Das kolloide Ion und das nicht dissoziierte Amylophosphat aben dann einen Druck von $36 \mathrm{~mm}$ Wassersăule aus, aus welchem sich beí einer Konzentration des Kolloids von 0,98 Proz. eine mittlere Molatgröße $M=62000$ berechnen läBt.

Wie die Tabelle II zeigt, wird bei der C. Neuberg'schen Phosphorylierung tatsāchlich Phosphorsäure in nicht dialysabler Form im Kolloid fixiert und erteilt der Substanz jene Eigenschaften, welche wir am nativen Amylopektin zu finden gewohnt sind: die Fähigkeit zur

1) Die Berechtigung dieser Annahme-folgt auch aus nachstehender Berechnung. $100 \mathrm{~g}$ der phosphorylierten Amylosen enthalten $2,19 \mathrm{~g} \mathrm{P}_{2} \mathrm{O}_{5}$. In der Voraussetzung, daB ein primăres Salz vorliegt, entsprăchen zwei Atomen Phosphor ein Atom $\mathrm{Ca}$ oder $2,19 \mathrm{~g} \mathrm{P}_{2} \mathrm{O}_{5} 0,615 \mathrm{~g} \mathrm{Ca}=0,0154$ Atome Kalzium. Unse re 0,98 prozentige Lơsung der phosphorylierten Amylosen müBte demnach bei völliger Absăttigung des ersten $\mathrm{H}$ - Ions der Amylophosphorsäure in bezug auf das. $\mathrm{Ca}$-Ion 0,000154 molekular sein. Aus der elektrischen Leitfăhigkeit berechneten wir dafür den Wert 0,0001255 .

2) In der V. Mitteilung (S. 144) und VIl. Mitteilung (S. 291) ist ein Druckfehler unterlaufen. Die Konstante für die Berechnung des osmotischen Drucks beträgt 231526 und nicht 231516 . 
Kleisterbildung, das Betragen eines typisch elektronegativen Kolloids, welches eine scharfe Abtrennung bei der Elektrodialyse ermöglicht, die Fällbarkeit durch Salze und die große Empfindlichkeit seiner Viskosität gegen die Reaktion der Lösung (Tabelle III).

Tabelle IlI.

Einflub von $\mathrm{NaOH}$ auf die innere Reibung.

\begin{tabular}{|c|c|c|c|c|}
\hline \multicolumn{2}{|c|}{ Normalkonzentration der Lauge } & - & $1.10^{-3}$ & $5.10-3$ \\
\hline & Natives Amylopektin & 2,79 & 3,21 & 3,68 \\
\hline 0,2 prozent. & Erythroamylosen . . . . & 1,052 & 1,034 & 1,040 \\
\hline Lösungen & $\begin{array}{c}\text { Phosphorylierte Erythroamy- } \\
\text { losen } . .\end{array}$ & 5,19 & 3,63 & 4,67 \\
\hline
\end{tabular}

Trotz aller Vorsicht erfolgte bei der Phosphorylierung eine partielle Desaggregation des Kolloids, so daß seine Molatgröße etwa auf die Hälfte des am nativen Amylopektin beobachteten Wertes gesunken ist. Da der Phosphorgehalt im hier beschriebenen Produkt mehr als zehnmal so groB ist wie im nativen Amylopektin, kommen auf ein osmotisch wirkendes Molat etwa 19 Molekule $\mathrm{H}_{3} \mathrm{PO}_{4}$. Nehmen wir mit P. Karrer') als Elementarmolekel der Stärkesubstanzen die Anhydromaltose an, so wäre das wirksame osmotische Molat im Mittel Jurch den Ausdruck $\left(\mathrm{C}_{12} \mathrm{H}_{20} \mathrm{O}_{10}\right)_{187}\left(\mathrm{H}_{3} \mathrm{PO}_{4}\right)_{19}$ gegeben $\left.{ }^{2}\right)$, während dem nativen Amylo-Pektin die Formel $\left(\mathrm{C}_{12} \mathrm{H}_{20} \mathrm{O}_{10}\right)_{348}\left(\mathrm{H}_{3} \mathrm{PO}_{4}\right)_{3}$ entsprechen würde.

Die bereits im geschilderten Versuche festgestellte Desaggregation der Molate führt während der Phosphorylierung um so weiter, je häufiger oder anhaltender infolge $z u$ langsamer Neutralisationswirkung des $\mathrm{CaCO}_{3}$ die Lösung sauer war.

Bei solcher Versuchsführung bleibt eine nicht unbeträchtliche Menge der neugebildeten Substanz gelöst, ein anderer. Teil befindet sich mit dem $\mathrm{CaCO}_{3}$ gemischt im Niederschlag und kann durch siedendes Wasser herausgelöst werden. Durch Alkoholfällung, Waschen mit wässerigem Alkohol und nachfolgende Elektrodialyse erhält man die

1) P. Karrer, C. Nägeli, O. Hurwitz u. A. Wälti, Helv. chim. Acta 4, 678 (1921).

2) Es braucht nicht betont $z u$ werden, daB die hier wiedergegebenen Formein nicht Ausdrücke für ein Molekel im Sinne der organischen Chemie bedeuten, sondern die stöchiometrischen Verhältnisse in dem angenommenen Molekuilaggregat wiedergeben sollen. 
Substanz rein in Lösung. Diese ist leicht opak und besitzt die aus Tabelle IV ersichtlichen Merkmale.

Tabelle IV.

\begin{tabular}{|c|c|c|c|c|c|}
\hline & $\begin{array}{l}\mathrm{P}_{2} \mathrm{O}_{\mathrm{b}} \\
\text { i. Proz. d. } \\
\text { Trocken- } \\
\text { substanz }\end{array}$ & $\begin{array}{l}10^{5} \mathrm{~K} \text { in } \\
2 \text { prozent. } \\
\text { Lösung }\end{array}$ & $\begin{array}{c}t / \dot{t}_{1} \text { in } \\
1 \text { prozent. } \\
\text { Loosung }\end{array}$ & $\begin{array}{c}\text { Mittlere } \\
\text { Molat- } \\
\text { grơBe }\end{array}$ & $\begin{array}{c}\text { Reduk- } \\
\text { tions- } \\
\text { vermơgen }\end{array}$ \\
\hline $\begin{array}{l}\text { In der Losg. gebliebener } \\
\text { Anteil } . \cdot{ }^{\circ} \cdot .\end{array}$ & 0,345 & 24,7 & 1,40 & 41200 & 0,58 \\
\hline $\begin{array}{l}\text { Aus d. } \mathrm{Ca} \mathrm{CO}_{3} \text {-Nieder- } \\
\text { schlage erhalt. Anteil }\end{array}$ & 0,244 & 17,7 & 1,47 & 28500 & 0,51 \\
\hline
\end{tabular}

Die stöchiometrischen Verhältnisse der beiden in dieser Tabelle beschriebenen Substanzen wären dann durch die Formel

für die lösliche und

$$
\left(\mathrm{C}_{12} \mathrm{H}_{20} \mathrm{O}_{10}\right)_{127}\left(\mathrm{H}_{3} \mathrm{PO}_{4}\right)_{2}
$$

für die unlösliche gegeben.

$$
\left(\mathrm{C}_{12} \mathrm{H}_{20} \mathrm{O}_{10}\right)_{88}\left(\mathrm{H}_{3} \mathrm{PO}_{4}\right)
$$

II.

Für die bisher beschriebenen Versuche dienten uns als Ausgangsmaterial die Erythroamylosen, das ist diejenige Substanz, welche von Natur aus mit Phosphorsäure verkoppelt auftritt.

Es ist theoretisch sehr interessant, dab es nach der geschilderten Methode auch möglich war, die Amyloamylosen zu phosphorylieren und daB das erhaltene Produkt in allen wesentlichen Eigenschaften mit dem aus den Erythroamylosen erhaltenen synthetischen Amylopektin übereinstimmt, mit dem Unterschiede, daB die phosphorylierten Amyloamylosen ihre rein blaue Jodfarbe beibehalten haben.

Die Phosphorylierung erfolgte in der bei der Elektrodialyse erhaltenen etwa 0,3 prozentigen Lösung und war innerhalb einer Stunde beendet. Die ursprünglich klare Lösung koagulierte während der Phosphorylierung, so dab zu Ende der Reaktion die Hauptmenge der Substanz in Form eines weißen flockigen Niederschlages ausgeschieden war. Im Gegensatz zu den Erythroamylosen erfahren die Amyloamylosen während der Phosphorylierung keine Erhöhung der inneren Reibung.

Das Reaktionsgemisch wurde in Pergamentsăcken unter Toluol gegen destilliertes Wasser, welches zweimal tăglich gewechselt wurde, dialysiert und nach elftägiger Dialyse elektrodialysiert. Während der 
Elektrodialyse sondern sich die weißen Flocken aus der Suspension ab, das entstehende Sol ist klar und gibt nach Jodzusatz nur eine geringe Menge tiefblauer Körner. Die abgeschiedenen Flocken sammeln sich zunächst an der Kathodenmembran an, werden im Laufe der Elektrodialyse durchsichtig, bewegen sich zur Anodenmembran und ballen sich daselbst zu einer außerordentlich zähen Gallerte zusammen. Das Produkt wurde in der üblichen Weise wiederholt mit Wasser gewaschen und dann durch Abnutschen ohne Filter von dem Waschwasser getrennt. Die so erhaltene Gallerte ist 1,72 prozentig, völlig steif und gibt mit Jod eine rein blaue Jodfarbe. Eine Uebersicht über ihre Eigenschaften, verglichen mit den Eigenschaften der Ausgangssubstanz enthalten die Tabellen $\mathrm{V}$ und Vl.

Tabelle V.

\begin{tabular}{|c|c|c|c|c|c|c|c|}
\hline & \begin{tabular}{|l}
$\mathrm{P}_{2} \mathrm{O}_{5}$ \\
i. Proz. \\
der \\
Trock-- \\
Subst.
\end{tabular} & $\left|\begin{array}{c}K .10^{5} \\
\text { in } \\
2 \text { proz. } \\
\left.\text { Lös. }^{*}\right)\end{array}\right|$ & $\mid \begin{array}{l}t / t_{1} \text { in } \\
1 \text { proz. } \\
\text { Lösg. }\end{array}$ & $\begin{array}{l}\text { Jod- } \\
\text { farbe }\end{array}$ & $\begin{array}{l}\text { Mittlere } \\
\text { Molat- } \\
\text { gröBe }\end{array}$ & $\begin{array}{l}\text { Dialysab- } \\
\text { ler Anteil } \\
\text { in Proz. }\end{array}$ & $\begin{array}{l}\text { Reduk- } \\
\text { tions- } \\
\text { ver- } \\
\text { mögen }\end{array}$ \\
\hline $\begin{array}{l}\text { Amyloamylosen aus } \\
\text { einer } 1 / 2 \mathrm{St} \text {. auf } 120^{\circ}\end{array}$ & & & & & & & \\
\hline $\begin{array}{l}\text { erhitzten } 2 \text { prozentig. } \\
\text { Stärkelösung }\end{array}$ & 0,00 & 0,2 & 1,008 & $\begin{array}{l}\text { rein } \\
\text { blau }\end{array}$ & 79300 & 0,65 & 0,3 \\
\hline $\begin{array}{c}\text { Dieselben nach der } \\
\text { Phosphorylierung }\end{array}$ & 2,06 & 91,3 & 43,1 & $\begin{array}{l}\text { rein } \\
\text { blau }\end{array}$ & 10300 & 6,8 & 0,5 \\
\hline
\end{tabular}

*) Bedeutung der Zeichen vgl. Tab. II.

Tabelle VI.

\begin{tabular}{|c|c|c|c|c|c|}
\hline \multirow{2}{*}{$\begin{array}{l}\text { Konzentration } \\
\text { der } \mathrm{NaOH} \text { : }\end{array}$} & \multicolumn{5}{|c|}{$t / t_{1}$ einer 0,07 proz. Lösung in Gegenwart von $\mathrm{NaOH}$} \\
\hline & 0 & $1.10^{-4} \mathrm{n}$ & $5.10^{-4} \mathrm{n}$ & $1.10^{-3} \mathrm{n}$ & $5.10^{-3} n$ \\
\hline Amyloamylosen & 1,006 & 1,007 & 1,007 & 1,008 & 1,009 \\
\hline $\begin{array}{l}\text { Phosphorylierte } \\
\text { Amyloamylosen }\end{array}$ & 3,78 & 3.21 & 2,52 & 2,01 & 1,40 \\
\hline
\end{tabular}

Die Berechnung der mittleren Molatgröße erfolgte ähnlich wie in Tabelle II unter Berücksichtigung der Konzentration der nicht dialysablen $\mathrm{H}$ - und $\mathrm{Ca}-\mathrm{lonen}$. Die Zusammensetzung des Molats gibt dieFormel $\left(\mathrm{C}_{12} \mathrm{H}_{20} \mathrm{O}_{10}\right)_{30}\left(\mathrm{H}_{3} \mathrm{PO}_{4}\right)_{3}$. 
III.

Wie aus den vorstehend beschriebenen Versuchen hervorgeht, gelingt es unter Zuhilfenahme einer bekannten Esterifizierungsmethode nicht gelatinierende Stärkeformen in gelatinierende überzuführen, während alle früheren Versuche, dies durch Zusatz von Säuren, Basen oder Salzen zu erreichen, fehlgeschlagen sind.

Zur Kontrolle ließen wir unter ganz analogen Bedingungen wie das Phosphoroxychlorid Natriumphosphat in gesättigter Lösung auf Amylosen einwirken. Eine erkleckliche Reihe solcher Versuche, welche bei verschiedenen Temperaturen durchgeführt wurden, gab stets dasselbe Resultat: durch Dialyse wandert das Salz wieder aus der Stärkelösung aus, die physikochemischen Merkmale der Lösung haben sich unter der Salzwirkung nicht verändert.

Hierdurch erscheint uns die Frage, ob die Phosphorsäure in der Stärke adsorptiv, als Komplex- oder als Estersãure gebunden ist, zu Gunsten der letzteren Annahme endgültig entschieden. Der Umstand, daB die Amylophosphorsäuren reaktionsfähige Wasserstoffionen besitzen, kann kein Gegenbeweis gegen die Esterbindung der Säure sein, wie H. Thryller ${ }^{1}$ ) sich das vorstellt, da bei Veresternng e in er Hydroxylgruppe der Phosphorsäure noch zwei reaktionsfähige $\mathrm{OH}$-Gruppen übrig bleiben. Die Estertheorie müBte erst dann fallen gelassen werden, wenn ein einwandfreier Beweis dafür erbracht worden wäre, dả die Amylophosphorsäure eine dreibasische Säure ist. Dies ist aber bisher nicht geschehen, es sprechen vielmehr alle unsere nunmehr schon recht zahlreichen Versuchsergebnisse für die Annahme einer zweiwertigen Amylophosphorsäure.

Die vorliegenden Beobachtungen über synthetische Amylophosphorsäuren bedeuten auch in der Frage der Stärke-Jod-Farbe einen gewissen Fortschritt.

Es hat an Stimmen nicht gefehlt, daß die Jodfarbe von dem physikochemischen Zustande der Stärkesubstanz abhängig sei und daB eben der gallertige Zustand des Amylopektins die Ursache für seine violette Jodfarbe sei. Weit entfernt davon, die Zustandsform einer Substanz als die Hauptbedingung ihrer Reaktionsfähigkeit leugnen zu wollen, haben wir stets die Ansicht vertreten, daB das verschiedene Verhalten einzelner Polysaccharide gegenüber Jod doch noch eine tiefere Ursache haben müsse. Es ist in diesem Zusammenhange sehr interessant, daß die Amyloamylosen durch Einführung von Phosphor-

1) H. Tryller, Chem.-Ztg. 44, 833 (1920). 
säure eine Substanz liefern, welche man ohne weiteres als Amylopektin ansprechen darf, welche aber mit Jod eine rein blaue Farbe gibt. Trotzdem sich also der Assoziationsgrad auf ein Siebentel verringert hat und die in Wasser zu einer völlig dünnen Flüssigkeit lösliche Substanz ein ausgesprochener Gallertbildner geworden ist, blieb die Jodfarbe unverändert.

Ebensowenig vermag die Verseifung des nativen Amylopektins sowie die Wiedereinführung der Phosphorsäure in die erhaltenen Erythroamylosen an der violetten Jodfarbe dieser Stoffgruppe etwas zu ändern. Daraus folgt, daB der Assoziationsgrad in den Grenzen von $M=150000$ und $M=2000$ für die Jodfarbe nicht bestimmend sein kann und daB ebensowenig die Jodfarbe von der Hydratation der Substanz abhängen kann. Es werden ferner die großen Divergenzen in den Angaben über die Jodfarbe des Amylopektins verständlich. Soferne man mit L. Maquenne als Amylopektin den kleisterbildenden Bestandteil der Stärke bezeichnet, kann es auf Grund unserer Versuche ein Jod bläuendes und ein Jod violett färbendes Amylopektin geben. Ist das Amylopektin aus Amyloamylosen zu sehr dicken Klumpen zusammengeballt, so dringt das Jod nur schwer in die Klumpen ein und färbt die Substanz nur äußerlich an. Es ist nicht ausgeschlossen, daB in der nativen Stärke auch die Amyloamylosen wenigstens teilweise mit Phosphorsäure verestert sind und $\mathrm{da} \beta$ je nach den Darstellungsbedingungen einmal das Erythro-, einmal das Amylo-Amylopektin erhalten wird. Einen wesentlichen Fortschritt in der Theorie des gallertigen $\mathrm{Zu}$ standes ermöglicht uns der Vergleich der einzelnen synthetischen Amylophosphorsäuren miteinander.

Das aus den Amyloamylosen dargestellte phosphorylierte Produkt zeigt von allen untersuchten Substanzen die größte Zähigkeit, trotzdem es nur eine Molatgröße von $M=10000$ besitzt. Das Verhältnis Phosphor: Maltoseanhydrid beträgt in dieser Substanz 1:10. Die entsprechenden phosphorylierten Erythroamylosen haben bei dem gleichen relativen $P$-Gehalte eine mittlere Molatgröße von $M=62000$ und liefern nur halb so zähe Gallerten wie die Amyloamylosen. Es ist demnach für die Steifheit einer Gallerte nicht nur die mittlere Molatgröße und der relative Gehalt am aufladenden Elektrolyten, sondern auch die Natur der organischen Grundsubstanz verantwortlich. Ueberlegen wir, daB die Amyloamylosen wenig löslich, zelluloseähnlich sind, die Erythroamylosen aber in Wasser leicht löslich und sehr lösungsstabil sind, so kommen wir zum Schlusse, daB eine Gallerte bei sonst gleichen Umständen um so steifer sein dürfte, je weniger 
losslich ihre Grundsubstanz ist, daß sie aber eine um so intensivere Aufladung benötigt, um in Gallertform überzugehen, je geringer ihre Lösungsstabilität ist. Von diesem Standpunkte aus wird uns die hohe Zähigkeit der Agargallerte verständlich, deren Grundsubstanz der Zellulose nahesteht, wenig lösungsstabil ist und erst durch Paarung mit Schwefelsäure ${ }^{1}$ ) eine gallertbildende Substanz liefert.

\section{Zusammenfassung.}

1. Durch Veresterung der aus dem Stärkekorn erhaltenen Erythroamylosen mit Phosphorsăure läBt sich ein dem Amylopektin völlig entsprechendes Produkt erhalten.

2. Auch die Amyloamylosen lassen sich durch Veresterung mit Phosphorsäure in eine dem Amylopektin analoge gallertbildende Substanz überführen.

3. Die Jodfarbe des phosphorylieren Produktes gleicht - vorausgesetzt, daß während der Veresterung keine wesentliche Veränderung der organischen Grundsubstanz erfolgt ist - der Jodfarbe des Ausgangsmaterials. Das Färbevermögen mit Jod ist daher vom Hydratationsgrade und dem Assoziationsgrade der Substanz in weiten Grenzen unabhăngig.

4. Es wurden die widersprechenden Angaben über die Jodfarbe des Amylopektins aufgeklärt.

5. Durch Vergleich einzelner phosphorylierter Stärkekohlehydrate miteinander ergaben sich neue Momente, welche zum Verstăndnis der Gallertbildung verwertet werden konnten.

1) M. Samec u. V. Isajevió, Compt. rend. 173, 1221 (1921). 